\title{
Alterações na Sensibilidade ao Contraste Relacionadas à Ingestão de Álcool ${ }^{1}$
}

\author{
Melyssa Kellyane Cavalcanti² \\ Natanael Antonio dos Santos \\ Universidade Federal da Paraíba
}

\begin{abstract}
RESUMO - O objetivo deste trabalho foi caracterizar a função de sensibilidade ao contraste (FSC) para freqüências espaciais de 0,25, 1,0 e 4,0 cpg (ciclos por grau de ângulo visual) na ausência (Grupo Controle-GC) e após a ingestão moderada de álcool (Grupo Experimental-GE). Para tanto, foi utilizado o método psicofísico da escolha forçada. Participaram do estudo quatro mulheres, de 21 a 30 anos, com acuidade visual normal ou corrigida. Os resultados mostraram diferenças significativas entre os grupos na freqüência de 4,0 cpg $(\mathrm{p}=0,039)$, sendo o GE mais sensível ao contraste do que o GC. Estes resultados sugerem alterações na FSC relacionadas à ingestão moderada de álcool.
\end{abstract}

Palavras-chave: percepção visual; sensibilidade ao contraste; ingestão de álcool; freqüência espacial; método psicofísico.

\section{Changes in the Contrast Sensitivity Related to the Alcohol Ingestion}

\begin{abstract}
The purpose of this paper was to characterize the contrast sensitivity function (CSF) for spatial frequencies equal to $0.25,1.0$ and $4.0 \mathrm{cpd}$ (cycles per degree) in the absence (Control Group-CG) and after moderate alcohol ingestion (Experimental Group-EG). For such, the forced choice psychophysical method was used. The participants were four 21 to 30 year-old-women with normal or corrected visual acuity. The results showed a significant difference between groups at the spatial frequency of $4.0 \mathrm{cpd}(\mathrm{p}=0.039)$, such that the EG was more sensitive to contrast than the CG. These results suggest alcohol-related alterations in the CSF.
\end{abstract}

Keywords: visual perception; contrast sensitivity; alcohol ingestion; spatial frequency; psychophysical method.

A função de sensibilidade ao contraste (FSC) é um indicador da percepção visual e uma das técnicas mais completas na avaliação teórica e clínica das funções visuais (Adams \& Courage, 2002; Richman \& Lyons, 1994; Van Sluyters, Atkinson, Held, Hoffman \& Shatz, 1990). Em estudos mais recentes, a FSC tem sido aplicada para avaliar várias alterações neuropatológicas, como a esclerose múltipla (Regan, Silver \& Murray, 1997), doenças de Alzheimer e de Parkinson (Polat, Sagi \& Norcia, 1997; Vleugels, Van, Lafosse, Ketelaer, \& Vandenbussche, 1998), esquizofrenia (Slaghuis \& Thompson, 2003) e a síndrome de Down (Suttle $\&$ Turner, 2004), pois permite avaliar objetivamente o funcionamento de mecanismos sensoriais básicos relacionados ao processamento visual de um objeto.

A FSC pode ser definida como o inverso da curva de limiar de contraste (1/FSC), que estima a quantidade mínima de contraste necessária para detectar um objeto com uma determinada freqüência espacial. O contraste é a relação entre a diferença da luminância máxima e luminância mínima dividido pela soma das duas. A freqüência espacial, por sua vez, corresponde ao número de ciclos (listras claras e escuras) por grau de ângulo visual (cpg) (Santos \& Simas, 2001).

1 Apoio Financeiro: CAPES e CNPq

2 Endereço: Rua Francisco Brandão, ${ }^{\circ}$ 513, Apt $^{\circ}$ 202, Edf. Antônio Francisco do Bú VIII, Bairro Manaíra. João Pessoa, PB. CEP 58038520. Tel: (83)3226-4476. E-mail: melyssa_cavalcanti@hotmail.com.
Estudos relacionando a ingestão moderada de álcool e a FSC são pouco explorados e apresentam resultados divergentes. Por exemplo, Andre (1996) investigou os efeitos do consumo de álcool com um nível de alcoolemia de 0,073\% BAC (blood alcohol concentration), sob duas condições de luminância $\left(12,9 \mathrm{~cd} / \mathrm{m}^{2}\right.$, cândelas por metro quadrado, e 0,129 $\mathrm{cd} / \mathrm{m}^{2}$ ), com estímulos móveis e estacionários, utilizando a FSC para freqüências espaciais de 1,5, 3,0 e 6,0 cpg. Todas as variáveis demonstraram efeitos interativos com o álcool e, no caso da FSC, houve uma redução significativa da sensibilidade na freqüência alta $(6,0$ cpg). Já Pearson e Timney (1998a) mensuraram a FSC para freqüências espaciais de $0,75,1,5$, $3,0,5,0,7,5$ e $10 \mathrm{cpg}$ em um nível de alcoolemia de 0,077 $\%$ BAC, e observaram que o álcool diminuiu a sensibilidade apenas nas freqüências espaciais altas (7,5 e $10 \mathrm{cpg})$.

Existem ainda estudos que investigam o efeito da desintoxicação alcoólica na FSC (Wegner, Günthner \& Fahle 2001), e a relação entre o alcoolismo e a FSC (Roquelaure \& cols., 1995). Entretanto, não foram encontrados estudos relacionando os efeitos da ingestão moderada de álcool à sensibilidade ao contraste, especificamente com mulheres não usuárias de álcool. Nesse sentido, o presente estudo procurou caracterizar respostas típicas do sistema visual para grades senoidais em mulheres na ausência (Grupo Controle - GC) e após a ingestão moderada de álcool (Grupo Experimental - GE). O objetivo principal foi descrever os efeitos da ingestão moderada de álcool na detecção de grades senoidais utilizando o método psicofísico da escolha forçada. 


\section{Método}

\section{Participantes}

Participaram do estudo quatro voluntários do sexo feminino, na faixa etária de 21-30 anos e com acuidade visual normal ou corrigida.

A participação na pesquisa ocorreu mediante a assinatura do Termo de Consentimento Livre e Esclarecido, conforme a resolução $n^{\circ}$ 196/96 do Conselho Nacional de Saúde. As participantes foram instruídas sobre o caráter da pesquisa, os critérios de anonimato e a possibilidade de recusar-se e/ ou retirar-se do estudo a qualquer momento sem prejuízo para ambas as partes. O trabalho foi registrado na Comissão Nacional de Ética em Pesquisa (CONESP/MS) com o número de protocolo 162/05.

\section{Equipamentos e estímulos}

Foram utilizados estímulos visuais acromáticos de freqüências espaciais (grades senoidais) de $0,25,1,0$ e 4,0 cpg para mensurar as curvas de sensibilidade ao contraste. Os estímulos foram gerados e apresentados em tons de cinza com diâmetro de aproximadamente 7 graus de ângulo visual, posicionados no centro da tela (Figura 1). Outras informações sobre estímulos elementares podem ser encontradas na literatura (Santos, Simas \& Nogueira, 2003, 2004).

Os estímulos foram gerados em um monitor de vídeo monocromático CLINTON MEDICAL de 21 polegadas, tela plana, digital, de alta resolução, controlado por um microcomputador. As medições foram feitas à distância de $150 \mathrm{~cm}$ da tela do monitor com visão binocular. A luminância média da tela foi de $0,7 \mathrm{~cd} / \mathrm{m}^{2}$ ajustada por um fotômetro do tipo Asahi Pentax $1^{\circ}$ Spot Meter.

A concentração de álcool no sangue foi estimada por um etilômetro, modelo BAF-110. A sessão experimental foi iniciada quando o teor de álcool alcançou o valor de $6 \mathrm{~g} / \mathrm{dl}$ (ou $0,06 \%$ BAC). A administração de álcool foi realizada sob a forma de Vodka com concentração de $38 \%$, diluída em suco de laranja natural sem açúcar na proporção de 1/1.

\section{Procedimento}

Os testes foram realizados no período da manhã, entre 10-12 horas. Todos os participantes foram orientados a não consumirem alimentos 3 horas antes do estudo e a não ingerirem álcool nas 24 horas que antecederam os testes (ainda assim, a concentração de álcool no sangue foi aferida antes do início do experimento). Momentos antes do teste, os participantes foram pesados em uma balança mecânica modelo TEC20 Techline e o volume alcoólico a ser ingerido foi calculado levando em consideração a massa corpórea, conforme a fórmula proposta por Drummer e Odell (2001): Cmax $(\mathrm{g} / \mathrm{l})=0,02 \times$ dose $(\mathrm{g}$ por $70 \mathrm{~kg}$ de peso corporal).

Foi utilizado um delineamento experimental com medidas repetidas, no qual todos os participantes foram testados em todas as freqüências e nas duas condições, antes da ingestão de álcool (GC) e após a ingestão de álcool (GE), em dias diferentes. A ordem em que cada voluntário passou por cada uma das duas condições (GC e GE) foi controlada pela técnica de contrabalanceamento. Essa técnica consiste apenas em dividir a amostra aleatoriamente pela metade, e testar a primeira metade da amostra inicialmente na condição controle e em seguida na experimental, e a segunda metade da amostra na ordem inversa.

As estimativas foram realizadas com método psicofísico da escolha forçada (Santos \& cols., 2003, 2004; Santos, Nogueira \& Simas, 2005). Esse método se baseia no cálculo da probabilidade de acertos consecutivos por parte do voluntário, ou seja, em cerca de 100 apresentações de escolhas entre os dois estímulos (estímulo de teste e estímulo neutro), o estímulo de teste é percebido $79 \%$ das vezes pelo voluntário.

O procedimento para medir o limiar para cada freqüência consistiu na apresentação sucessiva simples do par de estímulos, tendo o voluntário que escolher dentre eles qual continha a freqüência espacial em questão. $\mathrm{O}$ critério adotado para variar o contraste da freqüência de teste era o de três acertos consecutivos para decrescer uma unidade e um erro para acrescer da mesma unidade $(0,08 \%)$. A sessão experimental terminou automaticamente ao serem completadas seis reversões.
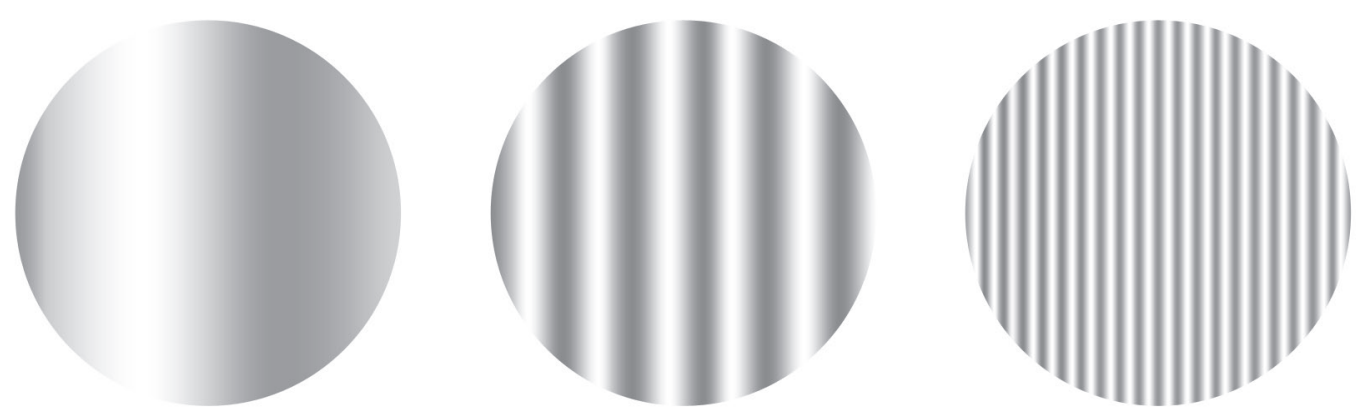

Figura 1. Exemplos de estímulos elementares grades senoidais com freqüências espaciais $0,25,1,0$ e 4,0 cpg, da esquerda para direita. Estímulos originalmente calibrados para serem vistos a $150 \mathrm{~cm}$ de distância antes de fotografados 


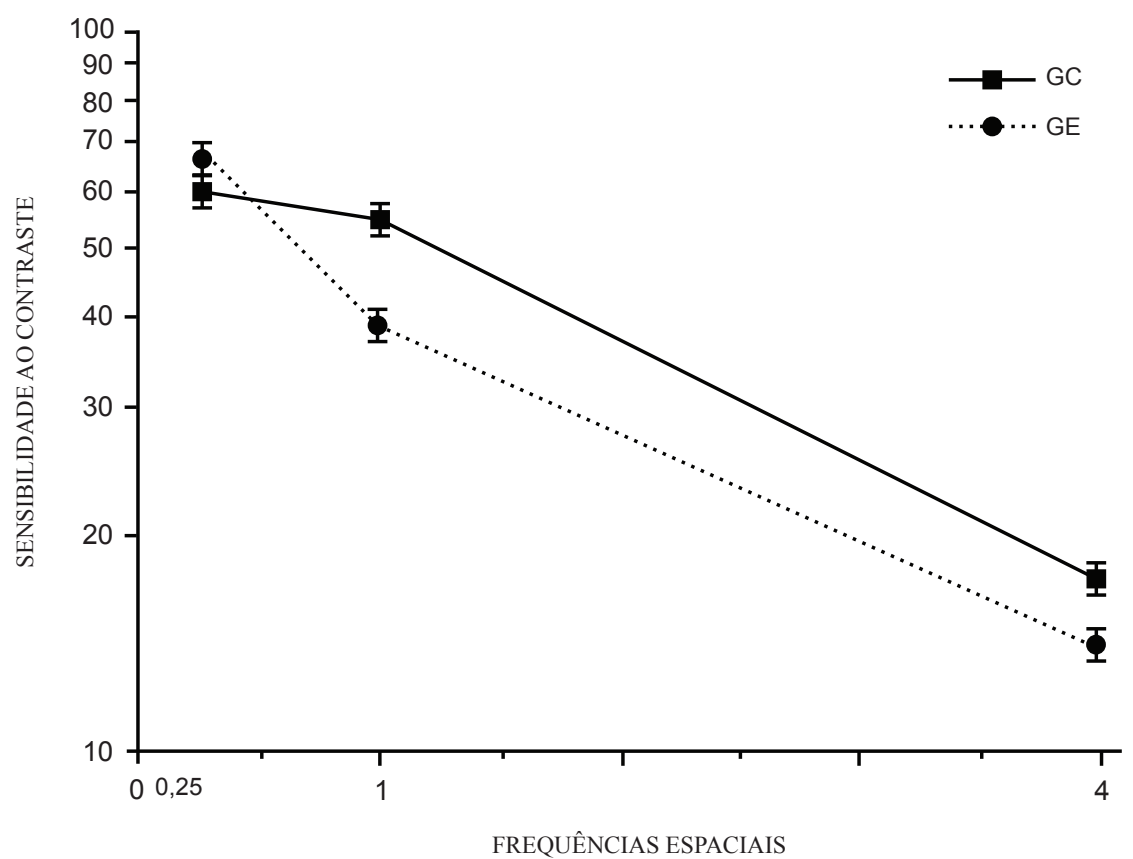

Figura 2. Curva de sensibilidade ao contraste de adultos do sexo feminino, sem (GC) e com (GE) ingestão moderada de álcool $(0,06 \% \mathrm{BAC})$. As linhas verticais mostram o erro padrão da média para cada freqüência espacial $(0,25,1,0$ e $4,0 \mathrm{cpg})$.

Os participantes foram orientados, antes da sessão, a pressionar o botão esquerdo do mouse ao julgarem que o estímulo de teste (freqüência espacial) tinha sido apresentado primeiro e o botão direito do mouse ao julgarem que o mesmo tinha sido apresentado em segundo lugar, isto é, após o estímulo de fundo. $\mathrm{O}$ estímulo de fundo era um estímulo neutro que correspondia a um círculo cinza homogêneo com luminância média de $0,7 \mathrm{~cd} / \mathrm{m}^{2}$. Assim sendo, a tarefa do voluntário foi escolher sempre o estímulo que continha uma das três freqüências espaciais de interesse $(0,25,1,0$ ou $4,0 \mathrm{cpg})$.

Os valores de contrastes máximos e mínimos obtidos para cada freqüência espacial foram agrupados em planilha por freqüência espacial e por grupo (GC e $\mathrm{GE}$ ) e, em seguida, aplicou-se a análise de variância (ANOVA) de dois fatores (Fator 1 - freqüência espacial em três níveis; Fator 2 - ingestão de álcool em dois níveis) para medidas repetidas, para comparar a sensibilidade ao contraste entre os grupos.

\section{Resultados}

A Figura 2 apresenta a média dos valores da sensibilidade ao contraste de todos os participantes em função das freqüências espaciais. As curvas de FSC dos participantes em ambos os grupos apresentaram maior sensibilidade na freqüência espacial mais baixa $(0,25 \mathrm{cpg})$ e menor sensibilidade na freqüência espacial mais alta $(4,0 \mathrm{cpg})$. No entanto, a FSC do GE foi da ordem de 1,14 vezes mais sensível do que a FSC do GC na freqüência de $0,25 \mathrm{cpg}$. Por outro lado, a FSC do GC foi da ordem de 1,04 e 1,27 vezes mais sensível do que a FSC do GE nas freqüências espaciais de 1,0 e 4,0 cpg, respectivamente.

A ANOVA para medidas repetidas mostrou efeito significativo de grupo $\left(F_{1,99}=9,25 ; p<0,01\right)$, de freqüência
$\left(\mathrm{F}_{2,188}=232,55 ; \mathrm{p}<0,01\right)$ e de interação de freqüência $\mathrm{x}$ grupo $\left(\mathrm{F}_{2,188}=5,05 ; \mathrm{p}<0,01\right)$. As análises com o teste post-hoc Tukey HSD mostraram diferenças significativas entre o GC e o GE apenas na freqüência de $4 \mathrm{cpg}(\mathrm{p}=0,039)$. Esses resultados indicam que a ingestão moderada de álcool altera a FSC na freqüência espacial de $4,0 \mathrm{cpg}$.

\section{Discussão}

Os resultados do presente estudo demonstraram que a FSC foi alterada pela ingestão moderada de álcool $(0,06 \%$ BAC). $\mathrm{O}$ álcool causou prejuízo significativo no processamento visual da freqüência espacial mais alta $(4,0$ cpg) e uma melhora não significativa no processamento visual da freqüência espacial mais baixa $(0,25 \mathrm{cpg})$. Resultados dessa natureza, em níveis baixos de contraste e com método psicofísico da escolha forçada, reforçam a idéia de que a ingestão moderada de álcool diminui a sensibilidade ao contraste nas freqüências mais altas (Andre, 1996; Pearson \& Timney, 1998a).

Esses resultados são semelhantes a outros relatados na literatura, que encontraram declínio da sensibilidade ao contraste em freqüências mais altas, em homens e mulheres, após o consumo de álcool, sob condições de luminâncias baixas de $21 \mathrm{~cd} / \mathrm{m}^{2}$ (Pearson, 1997; Pearson \& Timney, 1998b). Entretanto, os resultados do presente trabalho foram obtidos em condições metodológicas diferentes (luminância média de $0,7 \mathrm{~cd} / \mathrm{m}^{2}$ e sexo exclusivamente feminino).

Nesse sentido, os resultados deste trabalho corroboram a hipótese proposta por Pearson (1997) de que as vias paralelas magnocelular e parvocelular são afetadas de formas diferentes. A proposta de Pearson é de que a via visual dorsal (magnocelular), responsável pela mediação da percepção de movimento e profundidade, seria mais afetada pela ação 
do álcool quando comparada à via visual ventral (parvocelular), relacionada à percepção de contorno e contraste. As vias visuais podem ser acessadas por luminâncias médias diferentes. Ao utilizar uma luminância média $\left(0,7 \mathrm{~cd} / \mathrm{m}^{2}\right)$, provavelmente a via magnocelular foi acessada e, nessa condição, houve prejuízo na freqüência mais alta. Diante do exposto, infere-se que a ingestão moderada de álcool afeta o sistema visual humano de forma diferente.

Em termos gerais, estudos dessa natureza têm que levar em consideração algumas variáveis que podem confundir ou interagir com o efeito da ingestão de álcool, como por exemplo: controle da concentração alcoólica no sangue (Pearson \& Timney, 1998a; Figueira, 2002), período entre a última refeição e o experimento, o gênero, o uso do tabaco, a freqüência com que a pessoa bebe, dentre outros fatores (Drummer \& Odell, 2001; Edwards, Marshall \& Cook, 2003/2005).

Nesse contexto, o presente estudo investigou apenas o efeito da ingestão do álcool em adultos jovens do sexo feminino, não fumantes, e que raramente bebiam, porque as concentrações de álcool no sangue das mulheres são geralmente maiores do que nos homens, considerando-se a mesma quantidade consumida (Edwards \& cols., 2003/2005; Ramchandani, Bosron \& Li, 2001). Isso ocorre devido ao menor volume de água por peso corporal, à quantidade relativamente maior de gorduras e à menor atividade da enzima álcool-desidrogenase gástrica, enzima importante no metabolismo do etanol. Já a escolha da concentração de $0,06 \%$ BAC ocorreu devido este ser o limite máximo adotado pelo Código de Trânsito Brasileiro (1997). Por outro lado, novas pesquisas serão realizadas para avaliar o papel de outros níveis de luminância e o efeito placebo.

\section{Referências}

Adams, R. J. \& Courage, M. L. (2002). Using a single test to measure human contrast sensitivity from early childhood to maturity. Vision Research, 42, $1205-1210$.

Andre, J. T. (1996). Visual functioning in challenging conditions: Effects of alcohol consumption, luminance, stimulus motion, and glare on contrast sensitivity. Journal of Experimental Psychology, 2, 250-269.

Código de Trânsito Brasileiro (1997). Retirado em 02/01/2006 de http://www.senado.gov.br/web/codigos/transito/httoc.htm.

Drummer, O. H. \& Odell, M. (2001). Pharmacokinetics, metabolism and duration of action. Em O. H. Drummer \& M. Odell (Orgs.), The forensic pharmacology of drugs of abuse (pp.279-289). London: Arnold.

Edwards, G., Marshall E. J. \& Cook, C.C.H. (2005). O tratamento do alcoolismo: um guia para profissionais de saúde (4a ed.) (Miazzi, A.E.F, Trad.). Porto Alegre: Artmed. (Trabalho original publicado em 2003)

Figueira, I. (2002). Etanol e bebidas alcoólicas. Pode a actividade farmacológica do álcool explicar a diversidade de efeitos nos diferentes sistemas? Revista da Faculdade de Medicina de Lisboa, Série III, 7, 165-171.

Pearson, P. M. (1997). The effects of ethyl alcohol on visual and auditory thresholds. Tese de Doutorado, The University of Western Ontario, London, Ontario.

Pearson, P. \& Timney, B. (1998a). Alcohol does not affect visual contrast gain mechanisms. Visual Neuroscience, 16, 675-680.
Pearson, P. \& Timney, B. (1998b). Effects of moderate blood alcohol concentrations on spatial and temporal contrast sensitivity. Journal of Studies on Alcohol, 59, 163-173.

Polat, U., Sagi, D. \& Norcia, A. M. (1997). Abnormal long-range spatial interactions in amblyopia. Vision Research, 37, 737744.

Ramchandani, V. A., Bosron, W. F. \& Li, T. K. (2001). Research advances in ethanol metabolism. Pathologie-Biologie, 49, 676-682

Regan, D., Silver, R. \& Murray, T. J. (1997). Visual acuity and contrast sensitivity in multiple sclerosis-hidden visual class. Brain, 100, 563-579.

Richman, J. E. \& Lyons, S. (1994). A forced choice procedure for evaluation of contrast sensitivity function in preschool children. Journal of the American Optometric Association, $65,859-864$.

Roquelaure, Y, Gargasson, J. F. L. E., Kupper, S., Girre, C., Hispard, E. \& Dally, S. (1995). Alcohol consumption and visual contrast sensitivity. Alcohol \& Alcoholism, 30, 681-685.

Santos N. A. \& Simas, M. L. B. (2001). Função de Sensibilidade ao Contraste: indicador da percepção visual da forma e da resolução espacial. Psicologia Reflexão e Crítica, 14, 589-597.

Santos, N. A., Simas, M. L. B. \& Nogueira, R. M. T. B. L. (2003). Processamento visual da forma em idosos: Curvas de limiar de contraste para freqüências angulares e senoidais. Psicologia: Reflexão e Crítica, 16, 271-277.

Santos, N. A., Simas, M. L. B. \& Nogueira, R. M. T. B. L. (2004). Comparison of angular frequency contrast sensitivity in young and older adults. Brazilian Journal of Medical and Biological Research, 37, 375-378.

Santos, N. A., Nogueira, R. M. T. L. \& Simas, M. L. B. (2005). Processamento visual da forma: Evidências para canais múltiplos de freqüências angulares em humanos. Psicologia: Reflexão e Crítica, 18, 98-103.

Slaghuis, W. L. \& Thompson, A. K. (2003). The effect of peripheral visual motion on focal contrast sensitivity in positive - and negative - symptom schizophrenia. Neuropsychologia, 41, 968-980.

Suttle, C. M. \& Turner, A. M. (2004). Transient pattern visual evoked potentials in children with Down 's syndrome. Ophthalmic and Physiological Optics, 24, 91-99.

Van Sluyters, R. C., Atkinson, M. S., Held, R. M., Hoffman, K., \& Shatz, C. J. (1990). The development of vision and visual perception. Em S. W. Spillmann \& J. S. Werner (Orgs.), The neurophysiological foundations (pp. 349-379). New York: Academic Press.

Vleugels, L., Van N. A., Lafosse, C., Ketelaer, P. \& Vandenbussche, E. (1998). Temporal and spatial resolution in fovea vision of multiple sclerosis patients. Vision Research, 38, 2987-2997.

Wegner, A. J., Günthner, A. \& Fahle, M. (2001). Visual Performance and Recovery in Recently Detoxified Alcoholics. Alcohol \& Alcoholism, 369, 171-179.

Recebido em 30.05.2007

Primeira decisão editorial em 28.02.2008

Versão final em 15.03.2008

Aceito em 23.10.2008 